\title{
Evaluation of Central Fatigue by the Critical Flicker Fusion Threshold in Cyclists
}

Vicente Javier Clemente-Suárez, Montaña Diaz-Manzano

\begin{abstract}
Central fatigue related in sport stimuli wasstudied by Critical Flicker Fusion Threshold (CFFT) to know the influence of exercise on the central nervous system (CNS), but there is a lack of knowledge about the central or peripheral fatigue in endurance events. This study aimed to analyze changes in CFFT before and after the incremental cycle ergometer test as a means to assess the fatigue of the CNS. CFFT ascending and descending, the subjective criterion and sensory sensitivity were analysed in 8 professional male cyclists $(72.1 \pm 5.59 \mathrm{~kg}, 180.3 \pm 6.43 \mathrm{~cm}, 17.4 \pm 0.7$ years) before and after a maximum incremental cycle ergometer test (start 50w increases $50 \mathrm{w}$ each $5 \mathrm{~min}$ ). In this incremental test, the induced fatigue was probably more a peripheral phenomenon than one due to central nervous system fatigue, because sensory sensitivity did not significantly decrease. In conclusion, an incremental cycle ergometer test increased the cortical arousal and does not affect negatively the CNS of professional cyclists.
\end{abstract}

Keywords:

Cortical arousal, Central nervous system, Fatigue, Flicker fusion threshold, Cycle ergometer 